\title{
APUNTES CRÍTICOS Y ALGUNAS CONTRIBUCIONES DE LA EDUCACIÓN ESPECIAL A LA INCLUSIÓN EDUCATIVA
}

PhD. Rafael Félix Bell Rodríguez Instituto Tecnológico de Formación

rafael.bell@formacion.edu.ec
PhD Emma Zulay Delgado Saeteros Instituto Tecnológico de Formación zulayd@formacion.edu.ec

Palabras claves: educación especial, inclusión educativa, Recibido: 13 de febrero de 2017 diversidad.

Keywords: special education, educational inclusion, diversity.

Aceptado: 10 de marzo de 2017

\section{RESUMEN}

La inclusión educativa constituye uno de los temas generales de la educación de mayor actualidad y complejidad. El estudio de su relación con la Educación Especial, en cuyo seno se promovió su surgimiento, es una tarea de gran importancia pues brinda la posibilidad de analizar las principales limitaciones y contribuciones de ese denominado tipo de educación a fin de extraer los aprendizajes requeridos y propiciar, de esa manera, el indispensable avance en la concepción y el desarrollo de una educación de calidad para la diversidad del alumnado. Consecuentemente, los apuntes críticos y algunas de las contribuciones en torno a las cuales se reflexiona en el presente artículo, a partir de la revisión de los puntos de vista de diversos autores sobre el tema y de la propia actividad del autor en este campo, pretenden estimular la continuidad del debate al respecto, aportando ideas con el ánimo de promover el aprovechamiento de lo más valioso de la experiencia acumulada como un aporte para el logro de los fines.

\section{ABSTRACT}

Educational inclusion is one of the general themes of education with great complexity nowadays. The study of its relationship with Special Education- which promoted its creation in the first place - is a very important task. It offers the possibility of analyzing the main limitations and contributions of this so-called type of education in order to extract the required learning and to promote, in this way, the indispensable advance in the conception and development of the quality of education for student diversity. Consequently, the critical notes and some of the contributions reflected in this article- based on the review of the different authors' points of view on the subject as well as the author's own practice in this field- aim at stimulating the debate on this topic, offering ideas in order to promote the use of the most valuable of the accumulated experience as a contribution to the achievement of the goals. 


\section{INTRODUCCIÓN}

Con cierta frecuencia el análisis de muchos hechos, fenómenos y enfoques en el ámbito pedagógico y en la vida en general, se realiza sin hacer una valoración crítica integral de sus antecedentes y evolución. Esta situación se agrava con el surgimiento de nuevos modelos y orientaciones educativas, en cuyo caso, en ocasiones, priman los análisis centrados en las limitaciones de las experiencias, enfoques y prácticas anteriores, tal vez con la intención de que ello contribuya a exaltar, de mejor manera, el paso de avance que significa la propuesta que se presenta.

La relación entre la Educación Especial y la integración escolar primero y más recientemente su vínculo con la inclusión educativa no ha estado exenta de las tensiones que se derivan de la situación descrita, lo que no ha permitido revelar en toda su magnitud, las contribuciones de la Educación Especial a la inclusión educativa y sus potencialidades para favorecer un mayor avance hacia el cumplimiento de los propósitos de ese proceso.

Derivado de lo antes expuesto, el objetivo del presente artículo es aportar elementos que favorezcan el análisis crítico integral de la Educación Especial en relación con la inclusión educativa, resaltando, en ese contexto, tanto sus limitaciones como aquellas contribuciones cuyo aprovechamiento puede representar un aporte para el logro de los fines y la superación de los retos que la inclusión educativa plantea.

\section{DESARROLLO}

\section{Educación Especial: apuntes críticos}

La atención y la educación de las personas con algún tipo de discapacidad tiene una larga historia, en cuya trayectoria reciente pueden distinguirse, de acuerdo con Vicente y De Vicente (2006) las siguientes etapas: institucionalización, normalización e integración, que es el antecedente inmediato de la inclusión educativa.

Precisamente el surgimiento y desarrollo de este último enfoque tiene lugar a partir, entre otros factores, del análisis crítico de la Educación Especial, caracterizado según Vicente y De Vicente (2006), Yarza (2011) y Bell (2016) por los siguientes aspectos:

- Prioridad de los contenidos biológicos, clínicos y psicológicos de la discapacidad.

- Predominio de un complejo aparato conceptual con una fuerte orientación médica y rehabilitadora.

- Carácter sobreprotector y discriminatorio de diversas prácticas educativas y asistenciales.

- Elevados costos, limitado alcance y cuestionamiento de su efectividad.

- Desarticulación en relación con los sistemas educativos generales.

Las palabras de López Melero (2007) parecen ilustrar con nitidez estos apuntes críticos: "la cuestión se complica si pensamos que la educación (especial), influenciada por la medicina y la psicología, tiene que curar y no buscar nuevos sistemas de enseñanza" (p.10). 
Este mismo autor subraya lo siguiente:

Yo, como profesional de didáctica, deseo hablar de educación y no de terapia, y rechazo frontalmente el modelo pseudoeducativo que, como señala Margaret Wang, sirve para etiquetar a las personas y no para conocer cuál sería el sistema que nos permitiera conocer mejor y más adecuadamente cómo educar (p. 12).

A la luz de estos y otros planteamientos críticos, con el impulso del Movimiento de Educación para todos, de la adopción del modelo social de la discapacidad y de la proclamación de diversas declaraciones y convenciones nacionales e internacionales, en el mismo seno de la Educación Especial se generaron y desarrollaron, no sin contradicciones, diferentes propuestas y experiencias del amplio y heterogéneo enfoque actual de la inclusión educativa.

De esta manera, el surgimiento y la evolución de la inclusión educativa están indisolublemente ligados a la Educación Especial, cuyas críticas y contribuciones no han de ser subestimadas en el esfuerzo por desarrollar una educación de calidad que conduzca al éxito de todo el alumnado.

\section{La Educación Especial y el aprovechamiento de las potencialidades del cerebro}

De acuerdo con Campos (2010) "el cerebro es el único órgano del cuerpo humano que tiene la capacidad de aprender y a la vez enseñarse a sí mismo. Además, su enorme capacidad plástica le permite reorganizarse y reaprender de una forma espectacular, continuamente" (p. 6).

En relación con ello ya Vigotsky había señalado que:

Nuestra sustancia nerviosa es, con toda seguridad, la de mayor plasticidad de todas las que conocemos en la naturaleza. Por consiguiente, puede desarrollar, como ninguna otra, la capacidad para los cambios, para la acumulación de sus huellas y para la predisposición, las cuales constituyen la base de la memoria (p.221).

Precisamente, el aprovechamiento de esa marcada plasticidad del cerebro se convirtió en una de las premisas para el trabajo de la Educación Especial desde las edades más tempranas, lo que permitió obtener amplias evidencias acerca de cómo, bajo una influencia educativa adecuada y enriquecedora, los alumnos con alguna discapacidad son capaces de desarrollar mecanismos y respuestas creativas para la superación de las limitaciones u obstáculos en el camino hacia el logro de nuevos aprendizajes.

De este modo, la Educación Especial reforzó la importancia del conocimiento y aprovechamiento de la naturaleza y características del funcionamiento del cerebro para el aprendizaje humano, que se produce de diferentes maneras y por diferentes vías (Campos, 2010), una realidad que la inclusión educativa ha de considerar en toda su dimensión en su aspiración por alcanzar el éxito de todos los alumnos.

\section{Mediación pedagógica y su naturaleza transformadora}

Desde el punto de vista de su significado y de acuerdo con el Diccionario de la Real Academia de la Lengua, la mediación se define como la acción y el efecto de mediar y uno de los ámbitos con los que comúnmente se asocia su aplicación es el jurídico, 
en cuyo caso se relaciona con la labor que asumen determinadas personas en función de encontrar soluciones a situaciones en las que los intereses de sus representados se encuentran en conflicto.

Sin embargo, la definición e implicaciones de la mediación rebasan los límites del Derecho y se reflejan en disciplinas como la filosofía, de la que toma su origen en la Antigüedad, en la pedagogía y en sus diversas áreas de aplicación, entre las que sobresalen la escuela, la familia y los contextos laborales.

En el caso de la mediación aplicada al campo de la educación, en torno a la cual gira el contenido de este apartado, Grisales y González Agudelola (2010) la conceptualizan como "un proceso que establece puentes entre los contenidos y los sujetos del aprendizaje, para componer la comunicación entre las partes y posibilitar otras relaciones entre el contenido y los estudiantes" (p.128).

Para Labarrere (2008) la mediación pedagógica consiste en el desarrollo de determinados tipos de acciones y actividades destinadas a lograr modificaciones en las personas, por lo que este autor destaca como objetivo del referido tipo de mediación la transformación y la promoción de cambios en las personas tanto a nivel individual como colectivo.

Por su parte, Valverde (2009) apunta que "la mediación es una forma de interacción pedagógica que facilita el proceso de enseñanza y aprendizaje, en un contexto educativo participativo, creativo, expresivo y relacional" (p.6).

Más allá de los matices distintivos de cada una de las definiciones referidas, parecen no existir dudas para destacar el carácter activo, transformador y el fundamento comunicativo de la mediación, en cuya aplicación pedagógica se integran las dos modalidades esenciales de la mediación: la semiótica, en la que los símbolos juegan un papel preponderante y la mediación por modelación, en la que las personas interactúan de manera directa o con ayuda de determinados instrumentos (Labarrere, 2008).

Así, la interacción de los docentes con los alumnos con capacidades especiales y el desarrollo de la mediación en el contexto del aula y fuera de ella, permite constatar la naturaleza doblemente transformadora de la mediación, capaz de promover cambios en los alumnos y al mismo tiempo, generar transformaciones en el profesorado que se reflejan, entre otros, en los siguientes aspectos:

- Las actitudes que asumen en relación con la diversidad del alumnado y sus potencialidades para el aprendizaje.

- La comprensión del valor de la diversidad humana como una fuente para el enriquecimiento y la generación de nuevas experiencias de aprendizaje.

- El desarrollo de un mayor nivel de conciencia en relación con las necesidades de la población con discapacidad y las medidas a adoptar para favorecer su inclusión educativa y social.

- La incorporación de la diversidad y en particular de la discapacidad como uno de los temas para sus análisis y reflexiones personales y profesionales.

- El mejor conocimiento de sus características personales y emocionales y de sus capacidades para responder ante distintas situaciones, en ocasiones adversas, de la vida cotidiana.

Al hilo de lo señalado, el desarrollo de la inclusión educativa ha de favorecer la potenciación de la función mediadora de todos los docentes y de los propios alumnos, propiciando el logro de nuevas transformaciones en ellos, incluidas las 
señaladas, a fin de estimular el necesario avance hacia la inclusión, que es avanzar en función de la elevación de la calidad de la educación para todos.

\section{Valor metodológico de la Educación Especial}

La actividad educativa y todas las interacciones de los docentes con los escolares con alguna discapacidad y entre ellos mismos, aporta datos y conclusiones que pueden resultar claves para la solución de problemas psicológicos y pedagógicos generales.

Tal vez por esa razón Vigotsky (1982) en más de una ocasión acudió al estudio de casos y situaciones referidas a personas en las que se presentan diversas variedades cualitativas del desarrollo para profundizar en el análisis y la demostración de fenómenos generales. Por ejemplo, en su obra cumbre "Pensamiento y lenguaje", al abordar la investigación acerca del proceso de la formación de conceptos reflexionó acerca de lo que aporta el estudio del lenguaje en los niños sordos al análisis de la evolución general del pensamiento en complejos (Vigotsky, 1982).

A la luz de lo expuesto, el fundador del enfoque histórico-cultural ponderó el estudio de diversas alteraciones y procesos de desintegración de determinadas funciones y sistemas psicológicos como una vía para una mejor comprensión de los procesos psíquicos en general con el propósito de adoptar, en dependencia de sus características, las alternativas que favorezcan su mejor desarrollo y se conviertan en pautas para la prevención y determinación de las estrategias con una mayor efectividad y alcance.

Esta postura vigotskiana muestra su vigencia en la esfera del aprendizaje en la que las estrategias, las técnicas y los procedimientos que se han concebido y aplicado para la superación de las barreras a las que se enfrentan los estudiantes con determinada discapacidad resultan también de utilidad para otros estudiantes (Florian, 2014).

El dominio de las referidas estrategias enriquece el arsenal de los recursos educativos, didácticos y metodológicos de los docentes, potenciando su capacidad para el ajuste de la respuesta educativa a fin de brindar a la diversidad del alumnado una enseñanza que considere sus necesidades, motivaciones, intereses y potencialidades, una insoslayable premisa para el logro de la inclusión educativa.

En relación con el propósito antes enunciado, la comprensión del papel de las escuelas especiales y del talento humano formado en y para ese contexto y su relación con las instituciones del sistema educativo adquiere una singular connotación asociada a la necesidad de lograr que las referidas escuelas se conviertan en centros de recursos al servicio de otros centros educativos y de la comunidad y que, en los casos estrictamente necesarios, puedan brindar atención a aquellos escolares que presenten situaciones de marcada gravedad (Blanco, 2006).

\section{Carácter general de las leyes de la Pedagogía}

Por sus características y por su naturaleza multidisciplinaria y compleja, con cierta frecuencia se retoma el debate acerca de la definición de la Educación Especial como un campo de conocimiento o como una disciplina científica (López, 1997) al que se unen las tensiones que se generan cuando se intenta precisar las relaciones entre la ciencia pedagógica y la propia educación especial. 
En estas reflexiones se asume que no es posible comprender la educación especial al margen de la ciencia pedagógica y se comparte la posición expresada por Vigotsky (2001) al criticar los intentos por considerar la denominada educación especial como algo ajeno a la pedagogía. Al respecto precisó que:

Este criterio es profundamente erróneo y que esa extraterritorialidad le ha sido atribuida por error, en virtud de la natural incomprensión de fenómenos que aún no han sido investigados. Las leyes generales de la pedagogía sólo pueden ser leyes científicas en tanto sigan siendo igualmente aplicables a todos los terrenos de la educación (p.455).

En consonancia con ello, se impone la necesidad de empezar a cuestionar el argumento de que los maestros del sistema educativo general no cuentan con las habilidades que son requeridas para trabajar con los alumnos con necesidades educativas especiales, uno de los motivos utilizado con frecuencia para abogar por la adopción de medidas que no se corresponden con los propósitos de la inclusión educativa. (Florian, 2014).

El referido cuestionamiento implica la necesidad de la permanente formación de todo el profesorado, en la que resulta indispensable la incorporación de contenidos relacionados con la atención a la diversidad y la inclusión educativa pues nadie desconoce la existencia de ciertas particularidades en la educación de los niños ciegos, sordos, o de aquellos que presentan por ejemplo, una discapacidad intelectual, pero, como apuntó Vigotsky (2001) parece obvio que las diferentes formas y métodos de enseñanza y educación, aunque se ajusten a las leyes de la pedagogía, adquieren una orientación y determinadas especificidades en el trabajo con esos alumnos.

\section{Individualización y socialización en la educación}

El carácter colectivo e individual de la educación de la personalidad y su respeto (Addine, González y Recarey, 2002) es uno de los principios para la dirección del proceso pedagógico.

Como las referidas autoras apuntan, el proceso pedagógico debe concebirse y desarrollarse a partir del conocimiento de las características de cada estudiante, de su entorno familiar, social, de su estilo y ritmo de aprendizaje y de su desarrollo psicoevolutivo en general.

En ese sentido, no existen dudas de la contribución realizada por la Educación Especial al desarrollo y reconocimiento de este principio, considerando, en primer lugar, la prioridad que se le concede al conocimiento por parte del docente de las características propias de cada estudiante para en consecuencia, elaborar y aplicar las estrategias educativas más efectivas. Ello no debe conducir a interpretaciones erróneas, acerca de las cuales alertó Vigotsky (2001) al señalar:

Resulta por igual incorrecta la idea de que el problema de la individualización surge sólo con respecto a lo que trasciende los límites de la normalidad. Por el contrario, en cada niño tenemos algunas formas de individualización, si bien es cierto que no tan tajantemente subrayadas y expresadas como en la ceguera, la genialidad, la sordera o la oligofrenia. Pero un fenómeno no deja de ser el mismo si se reduce su expresión cuantitativa. La exigencia de la individualización de los recursos educativos, por lo tanto, también constituye una demanda general de la pedagogía y se extiende absolutamente a todos los niños (p.456). 
Esa exigencia, sin embargo, no puede soslayar el papel socializador que juega el grupo escolar, y el reto que presupone la conjugación de las ventajas de la individualización con el aprovechamiento de las posibilidades de la comunidad educativa como factor de aprendizaje (Cebeza, 2011).

Desde esa perspectiva, las mejores experiencias acumuladas en la Educación Especial pueden servir como punto de partida para impulsar el cumplimento de este principio y enriquecerlo en el contexto que la inclusión educativa genera y que ha de ser presidido por el pleno respeto a la diversidad y la generación de oportunidades para el aprendizaje exitoso de todo el alumnado.

\section{Diversidad e inteligencias múltiples}

Antes de la aparición y posterior desarrollo de la teoría de las inteligencias múltiples, en diversos entornos familiares, escolares y sociales se acumularon amplias evidencias empíricas que con el paso de los años se identificarían con la teoría formulada y desarrollada por Gardner (1983), cuya esencia, como precisan Suárez, Maiz y Meza (2010) "cuestiona las visiones tradicionales de la inteligencia porque se centran primordialmente en los aspectos cognitivos, descuidando el papel de la personalidad, las emociones y el entorno cultural en que se desarrollan los procesos mentales"(p. 86).

El predominio de esas visiones tradicionales repercutió de manera negativa durante mucho tiempo en la Educación Especial en la que, por otro lado, eran cada vez más palpables las manifestaciones de la presencia y del desarrollo en los estudiantes de diversos tipos de inteligencia, desde la lingüística hasta la musical (Gardner, 1983), constatándose el elevado aporte de las mismas al desarrollo de su personalidad.

En ese sentido, la contribución de la Educación Especial resulta incuestionable, pues sus objetivos y aspiraciones educativas en muchas ocasiones no quedaron únicamente limitados a aspectos puramente cognitivos, académicos o a los resultados de determinado test psicológico, sino que plantearon nuevas propuestas y se proyectaron con mayor integralidad, colocando, por ejemplo, el desarrollo emocional y su vínculo con el aprendizaje, como uno de sus pilares fundamentales, aportando, al mismo tiempo, un valiosísimo material para el desarrollo del principio pedagógico de la unidad de lo afectivo y lo cognitivo.

Lo escrito por Vigotsky (2001) resume con plena vigencia la esencia del referido principio: "la emoción no es una herramienta menos importante que el pensamiento. La preocupación del maestro debe consistir no sólo en que sus alumnos piensen detenidamente y asimilen la geografía, sino también que la sientan" (p. 184).

Como se desprende de lo arriba señalado y teniendo en cuenta los desafíos que la inclusión educativa deberá continuar superando se hace indispensable, como indica Campos (2010):

Considerar la filosofía de las Inteligencias Múltiples al esquematizar nuestro trabajo, al proponer diferentes aprendizajes o al programar las actividades que llevaremos a cabo en aula, permitirá que nuestros alumnos utilicen diferentes recursos (provenientes de sus múltiples inteligencias) para el aprendizaje y el desarrollo de capacidades (p. 7).

Consiguientemente es posible afirmar que del aprovechamiento de las potencialidades de las inteligencias múltiples en la diversidad del alumnado, de la disponibilidad de docentes comprometidos, emocionalmente inteligentes y dispuestos siempre a continuar formándose, capaces de generar y mantener, junto 
con sus alumnos, un clima de confianza, afecto, comprensión mutua y respeto, dependerá en gran medida la concreción de los objetivos de la inclusión educativa en el aula y más allá de sus paredes.

\section{CONCLUSIONES}

Las reflexiones realizadas en este artículo han permitido sintetizar los principales apuntes críticos en relación con la Educación Especial, en cuya esencia se encuentra la naturaleza clínico-rehabilitadora que prevaleció en este campo y que todavía está presente en diversas prácticas educativas.

Al respecto y en el marco de la inclusión educativa resulta también de mucho valor el análisis de las contribuciones de la Educación Especial esbozadas, en particular las referidas a:

$\checkmark$ El aprovechamiento de las potencialidades del cerebro.

$\checkmark$ La mediación pedagógica y su naturaleza transformadora.

$\checkmark$ El valor metodológico de la Educación Especial.

$\checkmark$ El carácter general de las leyes de la Pedagogía.

$\checkmark$ La individualización y socialización en la educación.

$\checkmark \quad$ La diversidad y las inteligencias múltiples.

Evidentemente, estos no son los únicos aportes de la Educación Especial a la inclusión educativa ni al desarrollo de la teoría pedagógica y psicológica general, pero en ellos y en su aplicación creativa a partir de las ideas de Vygotsky y de los diferentes autores consultados, la inclusión educativa con seguridad encontrará importantes puntos de referencia para guiar su labor y realizar una decisiva contribución a favor de una educación de calidad para todas y todos, una aspiración que la humanidad no debería continuar aplazando. 


\section{REFERENCIAS BIBLIOGRÁFICAS}

Addine, F.; González, A. y Recarey, S. (2002). Principios para la dirección del proceso pedagógico. En G. García (Comp) Compendio de Pedagogía (pp.80-101). La Habana: Editorial Pueblo y Educación.

Bell, R. (2016). Potencialidades de la inclusión educativa para la innovación en la Educación Superior. En J. Almuiñas, J. Galarza y J. López (Coord.) La innovación en la gestión universitaria. Experiencias y alternativas para su desarrollo (pp.191-205). Guayaquil: RED: DEES-ITB.

Blanco, G. (2006). La equidad y la inclusión social: uno de los desafíos de la educación y la escuela hoy. Reice: Revista Electrónica Iberoamericana sobre Calidad, Eficacia y Cambio en Educación, 4(3), 1-15.

Cabeza, A. (2011). Individualización del proceso de enseñanza-aprendizaje. Pedagogía Magna, 11, 8-13.

Campos, A. (2010). Neuroeducación: uniendo las neurociencias y la educación en la búsqueda del desarrollo humano. La Educación. Revista Digital, 143, 114. Recuperado de http://www.dragodsm.com/pdf/dragodsmneurociencias-educacion-y-desarrollo-06-2012.pdf

Florian, L. (2013). La educación especial en la era de la inclusión: ¿El fin de la educación especial o un nuevo comienzo? Revista latinoamericana de educación inclusiva, 7(2), 27-36.

Gardner, H. (1983). Frames of mind. New Yorks: Basic Books

Grisales, L. y González, E. (2010). De un modo de relacionar dos elementos contrarios a la mediación o acerca de la aproximación histórica al concepto mediación. Anagramas Rumbos y Sentidos de la Comunicación, 9(17), 117130. Recuperado

de https://scholar.google.es/scholar?q=de+un+modo+de+relacionar+dos+ele $\underline{\text { mentos+contrarios }}$

Labarrere, A. (2008). Bases conceptuales de la mediación y su importancia actual en la práctica pedagógica. Summa psicológica UST, 5(2), 87-96.

Recuperado de

https://scholar.google.es/scholar?q=Bases+conceptuales+de+la+mediaci\% C3\%B3n\&btnG $=\& h l=e s \& a s \_s d t=0 \% 2 C 5$

López, M. (1997). La educación (especial): ¿Hija de un dios menor en el mundo de la ciencia de la educación? Educar, 21, 7-17.

Suárez, J.; Maiz, F., y Meza, M. (2010). Inteligencias múltiples: Una innovación pedagógica para potenciar el proceso enseñanza aprendizaje. Investigación y Postgrado, 25(1), 81-94.

Valverde, M. (2009). La comunicación pedagógica: elemento transformador de la práctica educativa. Revista Electrónica "Actualidades Investigativas en Educación", 9(2), 1-18.

Vicente, A. y De Vicente, M. (2006). Una aproximación a la historia de la Educación Especial. Murcia: Diego Marín Librero Editor. 
Valverde, M. (2009). La comunicación pedagógica: elemento transformador de la práctica educativa. Revista Electrónica "Actualidades Investigativas en Educación", 9(2), 1-18.

Vicente, A. y De Vicente, M. (2006). Una aproximación a la historia de la Educación Especial. Murcia: Diego Marín Librero Editor.

Vygotsky, L. (1982). Pensamiento y lenguaje. La Habana: Pueblo y Educación.

Vygotsky, L. (2001). Psicología pedagógica. Un curso breve. Buenos Aires: Aique Grupo Editor.

Yarza, A. (2011). Corrientes pedagógicas, tradiciones pedagógicas y Educación Especial: pensando históricamente la Educación Especial en América Latina. Revista RUEDES, Red Universitaria de Educación Especial, 1(1), 321. Recuperado de http://tesis.udea.edu.co/dspace/handle/10495/3925 